\title{
BlogWall: Social and Cultural Interaction for Children
}

\author{
Adrian David Cheok, ${ }^{1}$ Owen Noel Newton Fernando, ${ }^{1}$ Janaka Prasad Wijesena, ${ }^{1}$ \\ Abd-ur-Rehman Mustafa, ${ }^{1}$ Ramkumar Shankar, ${ }^{1}$ Anne-Katrin Barthoff, ${ }^{1}$ Naoko Tosa, ${ }^{2}$ \\ Yongsoon Choi, ${ }^{1}$ and Mayank Agarwal ${ }^{1}$
}

\author{
${ }^{1}$ Mixed Reality Lab, National University of Singapore, Singapore 119077 \\ ${ }^{2}$ Academic Center for Computing and Media Studies, Kyoto University, Kyoto 606-8501, Japan \\ Correspondence should be addressed to Adrian David Cheok, adriancheok@mixedrealitylab.org
}

Received 11 October 2007; Accepted 11 August 2008

Recommended by Junichi Osada

Short message service (SMS) is extremely popular today. Currently, it is being mainly used for peer-to-peer communication. However, SMS could be used as public media platform to enhance social and public interactions in an intuitive way. We have developed BlogWall to extend the SMS to a new level of self-expression and public communication by combining art and poetry. Furthermore, it will provide a means of expression in the language that children can understand, and the forms of social communication. BlogWall can also be used to educate the children while they interact and play with the system. The most notable feature of the system is its ability to mix up and generate poetry in multiple languages such as English, Korean, Chinese poems, or Japanese "Haiku" all based on the SMS. This system facilitates a cultural experience to children unknowingly, thus it is a step into new forms of cultural computing.

Copyright (C) 2008 Adrian David Cheok et al. This is an open access article distributed under the Creative Commons Attribution License, which permits unrestricted use, distribution, and reproduction in any medium, provided the original work is properly cited.

\section{Introduction}

SMS or short message service is immensely popular among mobile phone users today [1]. The volume of short messages that Hong Kong people sent during the period December 2006 to November 2007 amounted to 3.28 billion, a record high since office of the telecommunications authority (OFTA) started collecting the figures in 2002, translating into an increase of $26 \%$ as compared to the same period in 2005 and 2006 [2]. But, it is primarily used for peer-to-peer communication. It has not widely being used for public or social communications.

BlogWall is an extension of the existing text messaging to a new level of self-expression and public communication, combining visual art and poetry. Furthermore, it provides a means of expression in the language that children can understand and the forms of social communication, which is an essential part of their lives. The application enables a person not only to express herself artistically, but also to entertain the masses in a form of digital graffiti. Figure 1 shows the concept design of BlogWall. The main feature of
BlogWall is its ability to mix-up poetry from different poems and to generate new poetry based on users' SMS.

System such as "i.plot" [3] discovers hidden connections between unrelated words by tracing possible paths through a database, traversing many two-word connections built from content based on publicly available resources. BlogWall extends the concept of "i.plot" further, creating a new type of poetry by elevating SMS to new art form. The longest words in the SMS are selected to locate synonyms from the Internet (currently by using the "Free dictionary website" [4]). The synonyms are used to rate poem lines out of a database. The highest-rated lines will be shown to the user. The extension of this poetry mode is the formation of cultural poetry. The system uses databases containing Korean, Chinese, and Japanese poetry and their English meaning. Synonyms found from the Internet are used to select poetry from these databases. The original text message, poetry in original language, and its English meaning are displayed on the screen.

Other than poetry mode, there are several other modes available in BlogWall. It can directly display users SMS in an 


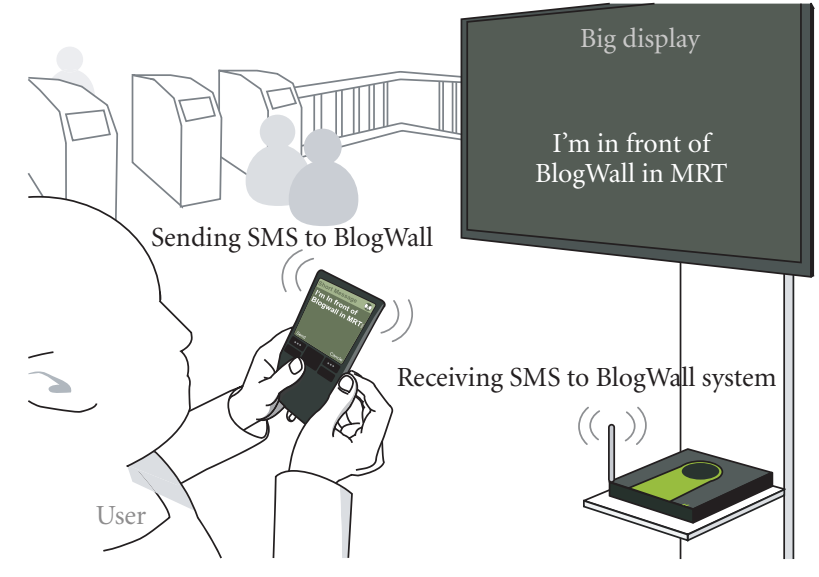

FIgURE 1: Concept design of BlogWall.

interesting way. The polling system available in BlogWall can be used to gather data from the public. Predefined keywords in the SMS can also be replaced by images when the SMS is displayed. Any of these modes can be activated based on users' requirements.

\section{Why Generate Poetry by SMS?}

People yearn to express themselves in public. Graffito is a one form of public expression that was very popular in mid70s [5]. With the growth of modern technology, people find new avenues to express themselves. Blogs enable people to share their ideas with millions of others all over the world. Today, Internet websites such as "Youtube" and "Twango" are popular ways of sharing videos and pictures with others.

Nowadays, electronic displays can be seen far and wide. These public displays are mainly used for conveying information rather than providing artistic and social communication which is of fundamental importance in the dubious world order. Modern life is weighted down not only by the global dilemmas, but also by the pace and the stress associated with it. Ironically, people spend a substantial amount of their precious time at airports, train stations, and so forth. One of the devices that almost all people have is a mobile phone. It will be a groundbreaking concept with unlimited possibilities ahead if artistic and social communication can be promoted using mobile phones and public displays.

Technological development poses undeniable threats because it will detach people from their cultures to a certain degree. Children of today who are interacting with these new technologies are finding more and more things to get absorbed in the popular culture. This development is affecting some of the cultural aspects of great cultures. Their folk songs, cultural dances, events, traditional dresses, and so forth are fast becoming museum pieces. But, it is not an inherent limitation of the technological development that is causing this, but it is how we use this new technologies. New technologies have successfully been integrated to preserve various cultures aspects. But, true preservation will not happen in a piece of plastic or silicon. It should happen in the hearts and minds of people. Since children are finding lot less in common with their culture, an approach must be created to embed culture into applications that children can understand and adore.

Poetry is one aspect of culture that has a long history, which will be recaptured in our system. It is also a wonderful way of expressing our inner thoughts. Studies have shown that poetry can be used very effectively to improve mental health [6]. Every one, in some manner, has a certain level of imagination and creativity to express himself in poetry. But, it is very hard for most of the people to actually construct a poem. Hence, a methodology should be there to bridge this gap effectively. SMS provides ideal basis because it is extremely user friendly, and since most people of today have mobile phones, it is a readily available facility.

\section{Related Research}

Researchers around the world have been experimenting with different combinations of art, public displays, and mobile messaging. The mobile phone has already been used as a medium of self-expression [7]. Ballagas et al. [8] discuss enabling interactions with large public displays using mobile phone. They have used the embedded camera on mobile phones as an enabling technology. Ballagas et al.'s "Point \& Shoot" technique allows users to select objects using visual codes to setup an absolute coordinate system on the display surface instead of tagging individual objects on the screen. Joe Blogg [9] is a public display, where users can contribute content by sending messages and images to it using their mobile phones. TexTales [10] is a large scale photographic installation to which people can send SMS text message captions. It can create technologically supported public discourse spheres in which they can both represent personal views and practice new ways of forming collective opinions.

One of the pioneering works in cultural computing was ZENetic computer [11]. It is an interface that evokes self-awakening through essential aspects of Zen Buddhist culture. It tries to offer users a chance to engage and understand Buddhist principles of "recreation" of the self. With stories portrayed in ink painting, haiku, and kimono, ZENetic conveys the rich allegorical interaction characteristic of Eastern philosophy. Another groundbreaking research was "Hitch Haiku" [12]. The system condenses an essence of a book into a Haiku, a Japanese minimal poem form. When a user chooses arbitrary phrases, system generates the haiku which includes the essence, then translates it into English. The cultural computing project ALICE [13] as an interactive, entertaining experience inspired from Alice in Wonderland [14]. In the scope of this project, interactive adventures are experiences provided by an augmented reality (AR) environment based on selected parts from Lewis Carroll's book Alice's Adventures in Wonderland. The user assumes the role of Alice and explores this interactive narrative. The project uses AR as a new medium for edutainment and entertainment as a particular carrier for cultural transformations.

The BlogWall consists of many of the features found on above-mentioned systems. SMS is the basis of BlogWall 


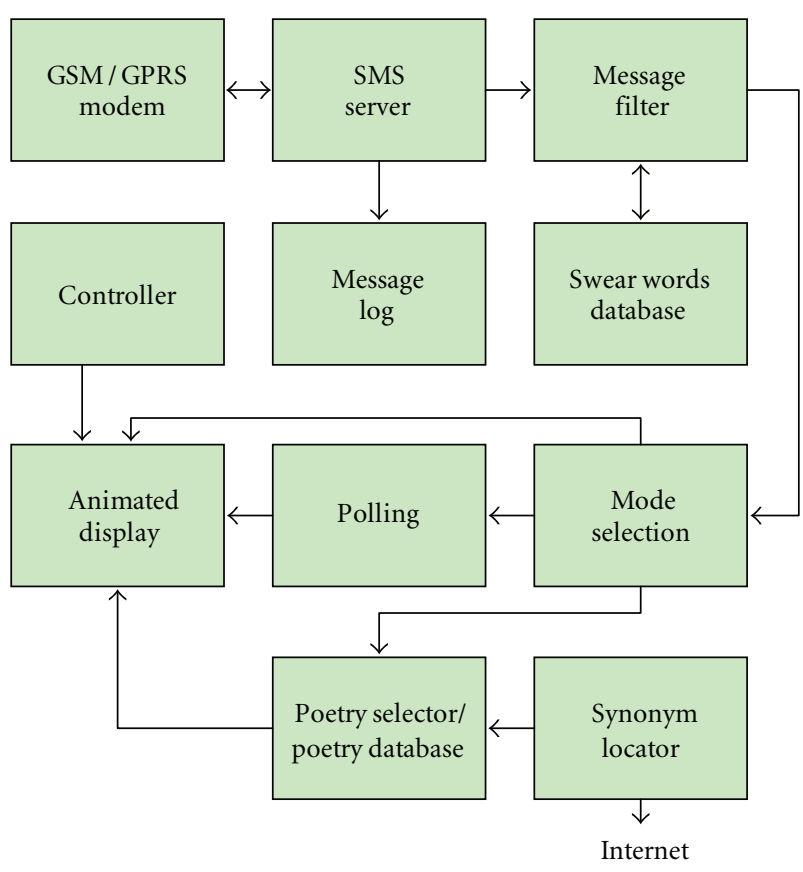

FIgURE 2: System overview.

operations like other systems. However, many of them directly display the SMS or use the mobile phone to control an action, but BlogWall uses the SMS to promote artistic and social communication through poetry. One of the main advantages of BlogWall over other cultural computing applications is its ease of use. Almost no domain knowledge is needed to use the BlogWall system. It has also been clearly designed to be used in public space by millions of users. Unlike other systems, using multilingual poetry, BlogWall can promote cross-cultural understanding.

\section{System Architecture}

The general setup of BlogWall requires a high-end computer with a good graphics card, projector, and a screen. Dedicated GSM/GPRS modem is used to receive SMS messages. The user stands in front of the projector screen and sends an SMS to a given number. The application issues AT commands to the modem to locate SMS. All the messages received by the server will be written to a log file along with originator phone number and the date/time. The application consists of several modes of operation. Based on the enabled modes, it offers different services to the user. The complete system architecture is shown in Figure 2.

\section{Display Modes}

Even though the main focus of BlogWall is poetry, there are several other ways it can be used. The additional modes of BlogWall can be activated based on user's requirements. These modes are mainly used as value addition to the overall system.

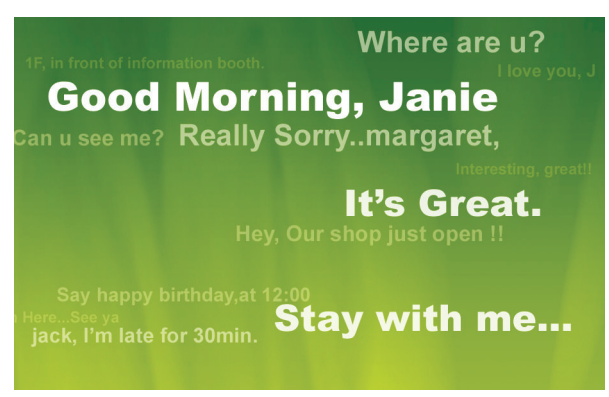

(a)

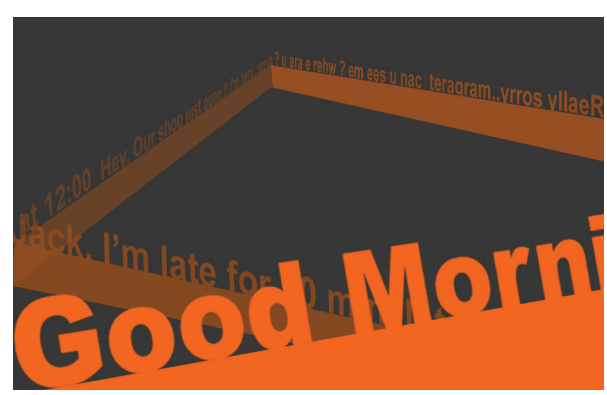

(b)

Figure 3: Animated display.

\subsection{Standard Display Mode}

If the application is in the standard display mode, it will immediately display the text message with some animation. When the system receives the SMS, it selects a random animation for the SMS as shown in Figure 3.

\subsection{Polling Mode}

The polling function is used to collect user opinions. The system displays polling question and available answers. The answers are indexed by single alphabetical letter within parenthesis. To vote, users send SMS with appropriate indexed letter of the answer to the system. BlogWall also has the capability to provide statistical data to system administrators.

\subsection{Keyword Triggering Mode}

Keyword triggering mode enables the application to trigger an internal function based on a word in the SMS. This feature is somewhat similar to the features found in popular chat programs like Windows Messenger. For example, if the SMS contains word "love" the application may replace the word "love" with an image of a heart. The keyword triggering mode can also display a small verse based on the words found in the SMS. The images as well as verses are selected from an internal database.

\subsection{Poetry Mode}

The most prominent feature of the application is its ability to mix poetry. In the poetry mode, user's SMS is analyzed 
to identify most prominent words. Those words are posted to a free dictionary website. The website will generate the synonyms related to the posted words, and the system finds them by analyzing the HTML response of the website. The synonyms are used to rank poems and poetry lines in an internal database. The database consists of lines of poetry and keywords related to each line and poem. When the system administrator adds a new poem to the system, all the keywords related to the poem and poem lines get generated and stored in separate table in the database.

The ranking of the poems is done based on the number of synonyms found in each poem. The poem that contains most number of synonyms gets the highest poem ranking. In the selected poem, the line that contains the highest number of synonyms gets the highest line ranking. Based on ranking, most suitable poetry lines will be selected to display.

For example, if the user sends an SMS, "I love thunder and rain" the selected words would be love, thunder, and rain. Some of the synonyms generated for word "love" may be passion, dearest, loved one. Based on the poem and line ranking, system might select the line "A heart whose love is innocent!" from the poem "She Walks in Beauty" by Lord Byron. Similarly, two more lines of poetry will be selected based on the synonyms generated for words thunder and rain. So, a possible output of this SMS might be the following:

\author{
"A heart whose love is innocent!" \\ "Boom, boom, boom," \\ "Rain from the clouds."
}

This unique ranking system enables the system to borrow lines of poetry from different poets. Therefore, the final outcome of the system could be unusual, surprising, or maybe amusing.

\subsubsection{Multilingual Poetry}

Due to the flexible nature of BlogWall, it can be extended to different cultures. The application has been extended to Korean, Japanese, Chinese, Malay, Tamil, and Sinhala poetry. The system consists of a very large poetry database. All the poetry in this database is translated to each of the displaying languages. When the system receives an SMS, it selects a line of poetry by ranking the English translation of it. The ranking system is similar to that of poetry mix-up method described above. Table 1 shows all the poetry generated for the SMS "I love thunder and rain" in multilingual poetry mode.

BlogWall system simultaneously displays the generated poetry in all different languages as shown in Figure 4. Each side of the rotating cube displays poetry in the respective language and its English meaning. Through the generation of multilingual poetry, we aim to create another step in the promotion of cross-cultural computing and cross-cultural understanding.
TABle 1: Poetry in multilanguages.

\begin{tabular}{|c|c|}
\hline Language & Poem \\
\hline \multirow{3}{*}{ English } & Rain of the cold season \\
\hline & Wing of love \\
\hline & The one of the thunder during the coldest season \\
\hline \multirow{3}{*}{ Haiku } & 寒の雨 \\
\hline & 愛の场根 \\
\hline & 寒雷の \\
\hline \multirow{3}{*}{ Chinese } & 寒之雨 \\
\hline & 爱的翅膀 \\
\hline & 寒雷之 \\
\hline \multirow{3}{*}{ Malay } & Hujan di musim sejuk \\
\hline & Sayab percintaan \\
\hline & Guruh di musim sejuk \\
\hline \multirow{3}{*}{ Tamil } & குளிர்கால மழை \\
\hline & காதலின் சிறகு \\
\hline & கடுங்குளிர்கால மின்னல்களில் ஒன்று \\
\hline \multirow{3}{*}{ Sinhala } & 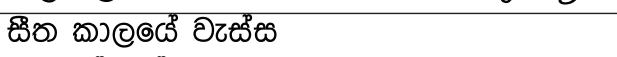 \\
\hline & 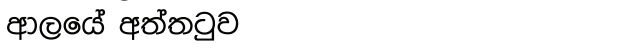 \\
\hline & 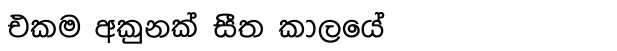 \\
\hline \multirow{3}{*}{ Korean } & 추운 계절의 비 \\
\hline & 사랑의 날개 \\
\hline & 가장 추운 계절의 번개 중의 하나 \\
\hline
\end{tabular}

\section{BlogWall User Study}

\subsection{Overview of the User Study}

The application has been demonstrated at several exhibitions and on permanent display at Singapore Science Centre (Figure 5). In those exhibitions, we have observed many people enjoying the interaction with BlogWall and we wanted to discover the interesting and exciting factors that attract the crowd towards this application. In this study, we focused on the social and engagement aspects of the user experience, while the cultural aspects will be a focus on future research.

To understand the users' engagement and social experience, we have used features described by Csikszentmihalyi's Flow theory [15]. The flow of the system is broken down as follows.

(1) Concentration. Since Concentration on the application is needed, the ability of the user to concentrate on the application is emphasized.

(2) Challenge. Does the system provide some level of challenge to the user.

(3) User Skills. User skill development and mastery should be supported in the system.

(4) Control. Users should be able to feel in control of their actions.

(5) Clear Goals. Clear defined goals should be given to the players at appropriate times.

(6) Feedback. Appropriate feedback from the system is given. 


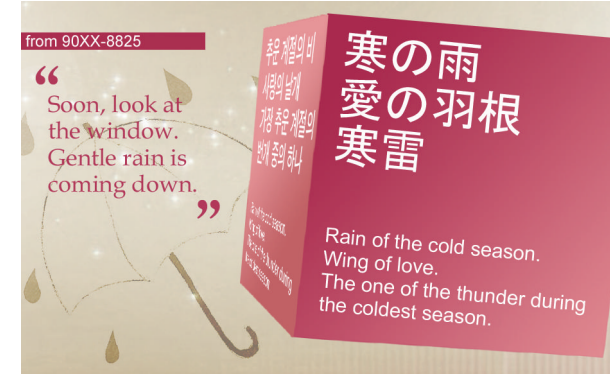

(a)

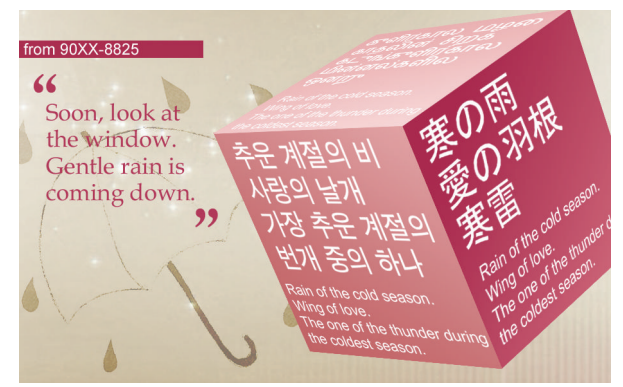

(b)

FIGURE 4: BlogWall poetry.

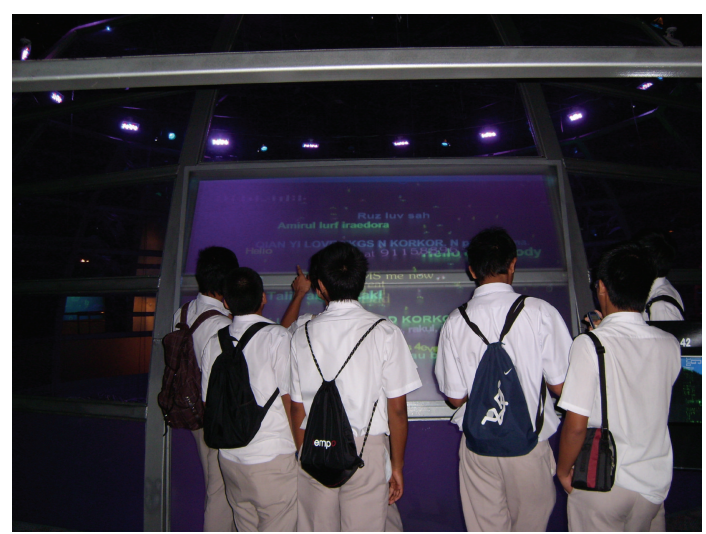

FIgURE 5: School children are interacting with BlogWall at Singapore Science Centre.

(7) Connection. Participants should feel deeply connected to the application and with little/no effort.

(8) Social Interaction. The system should support social interaction.

We came up with following set of questions to evaluate the system based on our hypotheses. Users were asked to interact with all the modes available in the system, including standard display, keyword triggering, and poetry. But, the main emphasis of the user study was focused on the poetry mode.

\subsection{User Study Questions}

User study questions and hypothesis pairs are as follows.

\section{(1) User Concentration of the System}

Question. Did the application managed to capture users' attention?

Hypothesis. The application should be able to capture users' attention.

Example Question. (1) Did the application grab your attention and maintain your focus?

Question. Were they able to concentrate on the task?

Hypothesis. User should be able to concentrate on the task at hand without any difficulties.

Example Question. (2) Were you able to concentrate on the tasks at hand?

\section{(2) User Skills Required in the System}

Question. Were the users able to interact with the system without much assistance?

Hypothesis. The only required skill expected in the system is the ability to send SMS. So the users should be able to proceed with little instructions.

Example Question. (3) Were you able to use the system without spending too much time at the instructions?

Question. Was learning to use the system fun?

Hypothesis. Time spent on learning to operate the system is small. Few simple instructions would be sufficient to start interacting with BlogWall. Still learning how to create poetry from SMS should be fun.

Example Question. (4) Was learning to use the system fun?

\section{(3) Amount of Control Users Has of the System}

Question. Did users feel they were in control instead of the system?

Hypothesis. The system behavior is directly connected to users' actions. So they should feel very much in control.

Example Question. (5) Did you feel in control of your actions in the system?

Question. Did they have control of the poetry output of the system?

Hypothesis. The poetry is generated using the SMS send by the user. BlogWall make use of the synonyms it found to generate poetry. Final output may be a surprising result to the users SMS. However, it is still derived from users SMS. Therefore, they are very much in control of the poetry output. 
Example Question. (6) Did you feel you were in control of the poetry output of the system?

\section{(4) Clear Goal Is Given to the Users}

Question. Was the overall objective of the system is clear to the user?

Hypothesis. Just by looking at the system, users should be able to comprehend the goal of the system.

Example Question. (7) Was the objective of the system clear and presented in advance?

\section{(5) Feedback Given to the Users by the System}

Question. Did users feel they knew what is happening in the system all the time?

Hypothesis. Users should have a general notion of what is happening inside the system. But, they may not have a complete image of how the system is progressing.

Example Question. (8) Were you aware what is happening in the system at all times?

Question. Did the system provide fast feedback?

Hypothesis. The system is based on SMS. The time required to receive the SMS should be very small. But, it might take some time to generate poetry since it has to search the Internet for the synonyms. Still the feedback of the system should be very high.

Example Question. (9) Did the system give you immediate feedback of your actions?

\section{(6) How Connected Are the Users to the System}

Question. Were users deeply involved in interacting with the system?

Hypothesis. The main task for the user is sending an SMS. But to generate proper poetry, they have to think deeply about the SMS. Therefore, there should be a medium level of connection with the system.

Example Questions. (10) During participation, were you less aware of what is happening physically around you?

(11) Were you aware of the passing of time during participation?

Question. Were they emotionally involved with the system?

Hypothesis. Aesthetic creativeness is very much an emotional process. They may be emotionally involved with the creative process of poetry thus inadvertently absorbed with the system as their interaction is with the system. Users should be emotionally involved with the system.
Example Question. (12) Did you feel emotionally involved with the system?

\section{(7) Social Interaction of the System}

Question. Did it support social interaction?

Hypothesis. One of the main objectives of the system is to support social communication. Large number of people can interact with the system enabling social communication. Therefore, users should feel the application supporting social communication.

Example Question. (13) Do you suppose that the system support social communication?

Question. Does the system support social communities inside and outside the system?

Hypothesis. Generally, users are expected to assemble to where the BlogWall is setup in order to interact. However, users can also send SMS to the system and interact with it without physically being there. Therefore, they should feel BlogWall does support social communities.

Example Question. (14) Does the system support social communities inside and outside the system?

The original set of questions for the flow model has been created for a generic task (like surfing the Internet), therefore, some questions have been modified to adapt to the BlogWall system. For the user study, 13 subjects were randomly selected and their average age is 27.3 (60\% male and $40 \%$ female).

\subsection{Results of the User Study}

Data collected from the survey after using the system are expressed as mean and standard deviation unless otherwise specified. Results of the survey are given in Table 2. Of all elements explored with this survey, most of them performed positively in the survey as more than 50\% selected the favorable choice to the questions posed.

Unlike in computer games, users do not have to concentrate very hard on the task at hand. Users can take their time in interacting with the system. It attracted the users but does not demand total concentration. This may explain the answers received for questions 1 and 2 .

One of the reasons we chose SMS as the basis for the poetry generation is that, it is very easy to use and according to the results of question 3,69\% users highly agree to that.

The overwhelming majority, 61\%, responded positively to question 6. Thus, users clearly feel that they can control the poetry output of the system. However, they did not respond very positively to question 5 . This means that users did not feel that they are in total control of their actions. The poetry generation does not depend totally on users SMS. BlogWall searches the Internet and finds synonyms for the selected words in the SMS. Therefore, the final poetry may 
TABLE 2: Results of user study.

\begin{tabular}{lccccc}
\hline Qn & Yes very & Yes & $\begin{array}{c}\text { Options } \\
\text { Fairly }\end{array}$ & Not really & No \\
\hline 1 & $8 \%$ & $70 \%$ & $22 \%$ & $0 \%$ & $0 \%$ \\
2 & $31 \%$ & $46 \%$ & $15 \%$ & $8 \%$ & $0 \%$ \\
3 & $69 \%$ & $31 \%$ & $0 \%$ & $0 \%$ & $0 \%$ \\
4 & $16 \%$ & $47 \%$ & $37 \%$ & $0 \%$ & $0 \%$ \\
5 & $16 \%$ & $39 \%$ & $45 \%$ & $0 \%$ & $0 \%$ \\
6 & $61 \%$ & $28 \%$ & $11 \%$ & $0 \%$ & $0 \%$ \\
7 & $0 \%$ & $53 \%$ & $31 \%$ & $8 \%$ & $8 \%$ \\
8 & $8 \%$ & $45 \%$ & $39 \%$ & $8 \%$ & $0 \%$ \\
9 & $31 \%$ & $54 \%$ & $15 \%$ & $0 \%$ & $0 \%$ \\
10 & $0 \%$ & $24 \%$ & $52 \%$ & $24 \%$ & $0 \%$ \\
11 & $0 \%$ & $24 \%$ & $45 \%$ & $31 \%$ & $0 \%$ \\
12 & $16 \%$ & $31 \%$ & $29 \%$ & $24 \%$ & $0 \%$ \\
13 & $47 \%$ & $39 \%$ & $14 \%$ & $0 \%$ & $0 \%$ \\
14 & $24 \%$ & $52 \%$ & $24 \%$ & $0 \%$ & $0 \%$ \\
\hline
\end{tabular}

not contain the exact words in the users SMS. In fact, this is a feature of BlogWall. But, it may explain the users' surprise of the outcome and the feeling of not totally being in control.

There is a clear indication that some users considered that the objective of the system is not clearly portrayed. $16 \%$ of the users responded negatively to question 7 . One user commented that "short instruction information should be displayed along with the BlogWall." Based on these findings, we are now displaying clear instructions to the users wherever we install the BlogWall system. Short description is also presented on the BlogWall display.

Even though feedback time can be compromised by mobile phone network and Internet delays, 31\% of users do agree that the system provides an immediate response. This means that the users feel that the system response time is satisfactory. There is a time gap between user sending the SMS and system receiving it. After receiving the SMS, the system searches the Internet to locate the synonyms. During those processes, there is no indication to the user that the system is processing her SMS, which may be the reason that $8 \%$ responded that they were unaware of the activities of the system at all times.

Only 24\% were less aware of what is happening around them and $24 \%$ were aware of the passing of time during participation. This is not an unexpected result since BlogWall does not require the user to be fully mentally connected with it. The response to question 12 is disappointing, $24 \%$ users felt that there is no emotional connection with the system. This can be explained by the fact that the poetry selection algorithm selects poetry based on simple line ranking. The selected poem may not reflect the overall context of the users SMS. By developing a natural language processor, the system should be able to analyze the context or linguistic meaning of the SMS and generate the poetry accordingly.

None of the users responded negatively to questions 13 and 14. This indicated that people did feel that the system does support social communication. Also, they believed it supports social communities inside and outside the system.
This is a very significant result since social interaction is one of the foremost aspirations of BlogWall. According to the user response, BlogWall has successfully achieved it.

\section{Conclusions}

BlogWall is a novel mobile artistic media application which promotes self-expression and public communication, combining visual art and poetry. By selecting proper elements, the application can be effectively used to build awareness among children about diverse cultural elements of humans as global beings. BlogWall can also be used for other purposes, such as learning applications. For instance, students and teachers could use the BlogWall to ask questions and provide answers in real-time. The users studies conducted indicate that the application has a high level of social and engagement aspects.

\section{Acknowledgments}

The initial version of the BlogWall is funded by Singapore Science Centre and it is installed in the iSpace at Singapore Science Centre.

\section{References}

[1] R. E. Grinter and M. A. Eldridge, "y do tngrs luv 2 txt msg?" in Proceedings of the 7th European Conference on Computer Supported Cooperative Work (ECSCW'01), pp. 219238, Kluwer Academic Publishers, Bonn, Germany, September 2001.

[2] Hong Kong SMS Usage Increased, 2007, http://www.chinatechnews.com/2008/02/01/6361-hong-kong-sms-usage-increased-in-2007.

[3] N. Tosa, A. R. Torroella, B. Ellis, S. Matsuoka, and R. Nakatsu, "Computing inspiration: i.plot," in Proceedings of the 32nd International Conference on Computer Graphics and Interactive Techniques (SIGGRAPH '05), p. 3, ACM Press, Los Angeles, Calif, USA, July-August 2005.

[4] Free dictionary, http://www.thefreedictionary.com/.

[5] "Graffiti," http://en.wikipedia.org/wiki/Graffiti.

[6] S. McArdle and R. Byrt, "Fiction, poetry and mental health: expressive and therapeutic uses of literature," Journal of Psychiatric \& Mental Health Nursing, vol. 8, no. 6, pp. 517-524, 2001.

[7] K. Cheverst, A. Dix, D. Fitton, et al., "Exploring mobile phone interaction with situated display," in Proceedings of Workshop on Pervasive Mobile Interaction Devices (PERMID '05), pp. 4347, Munich, Germany, May 2005.

[8] R. Ballagas, M. Rohs, and J. G. Sheridan, "Sweep and point and shoot: phonecam-based interactions for large public displays," in Proceedings of Conference on Human Factors in Computing Systems (CHI '05), pp. 1200-1203, ACM Press, Portland, Ore, USA, April 2005.

[9] K. Martin, A. Penn, and L. Gavin, "Engaging with a situated display via picture messaging," in Proceedings of Conference on Human Factors in Computing Systems (CHI '06), pp. 10791084, ACM Press, Montréal, Canada, April 2006.

[10] M. Ananny, C. Strohecker, and K. Biddick, "Shifting scales on common ground: developing personal expressions and public opinions," International Journal of Continuing Engineering 
Education and Life-Long Learning, vol. 14, no. 6, pp. 484-505, 2004.

[11] N. Tosa, S. Matsuoka, and K. Miyazaki, "Interactive storytelling system using behavior-based non-verbal information: ZENetic computer," in Proceedings of the 11th ACM International Conference on Multimedia (MULTIMEDIA '03), pp. 466-467, ACM Press, Berkeley, Calif, USA, November 2003.

[12] N. Tosa, "Hitch-Haiku," in Proceedings of the 34th International Conference on Computer Graphics and Interactive Techniques (SIGGRAPH '07), p. 250, ACM Press, San Diego, Calif, USA, August 2007.

[13] R. Nakatsu, M. Rauterberg, and P. Vorderer, "A new framework for entertainment computing: from passive to active experience," in Proceedings of the 4th International Conference on Entertainment Computing (ICEC '05), vol. 3711 of Lecture Notes in Computer Science, pp. 1-12, Sanda, Japan, September 2005.

[14] L. Carroll, Alice's Adventures in Wonderland, Macmillan, London, UK, 1865.

[15] M. Csikszentmihalyi, Beyond Boredom and Anxiety: Experiencing Flow in Work and Play, Jossey-Bass, San Francisco, Calif, USA, 1975. 

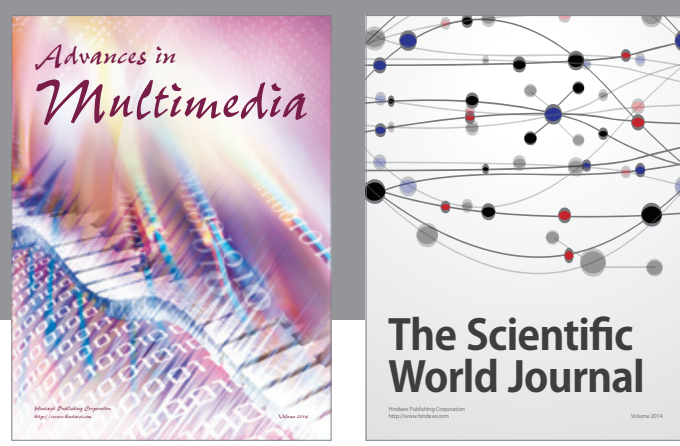

The Scientific World Journal
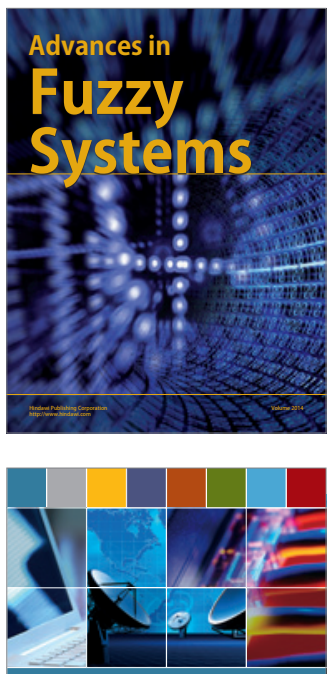

Computer Networks and Communications
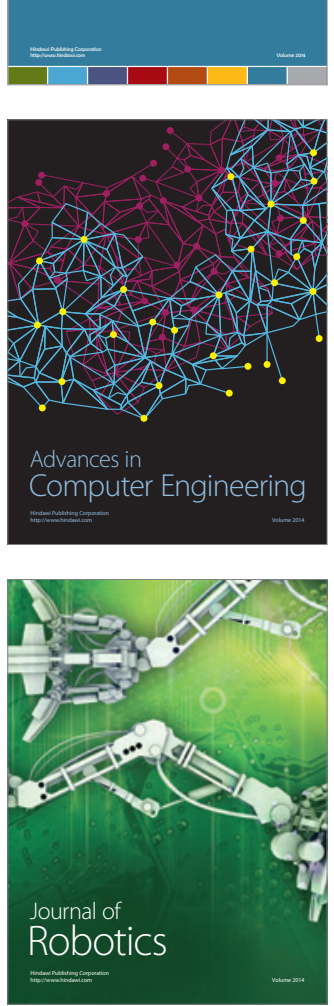
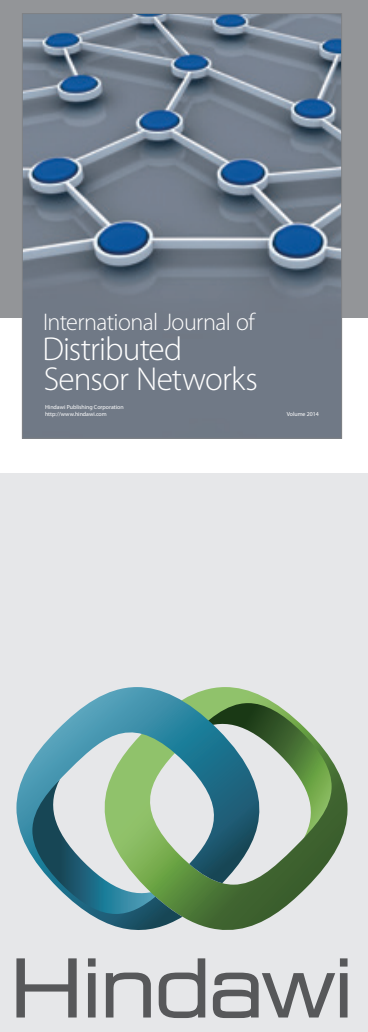

Submit your manuscripts at

http://www.hindawi.com
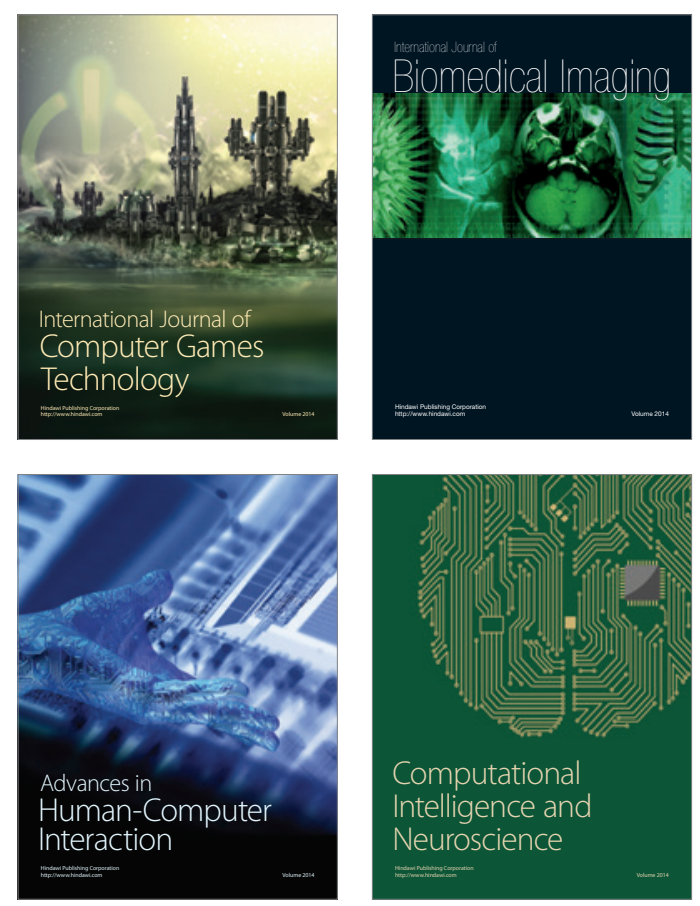
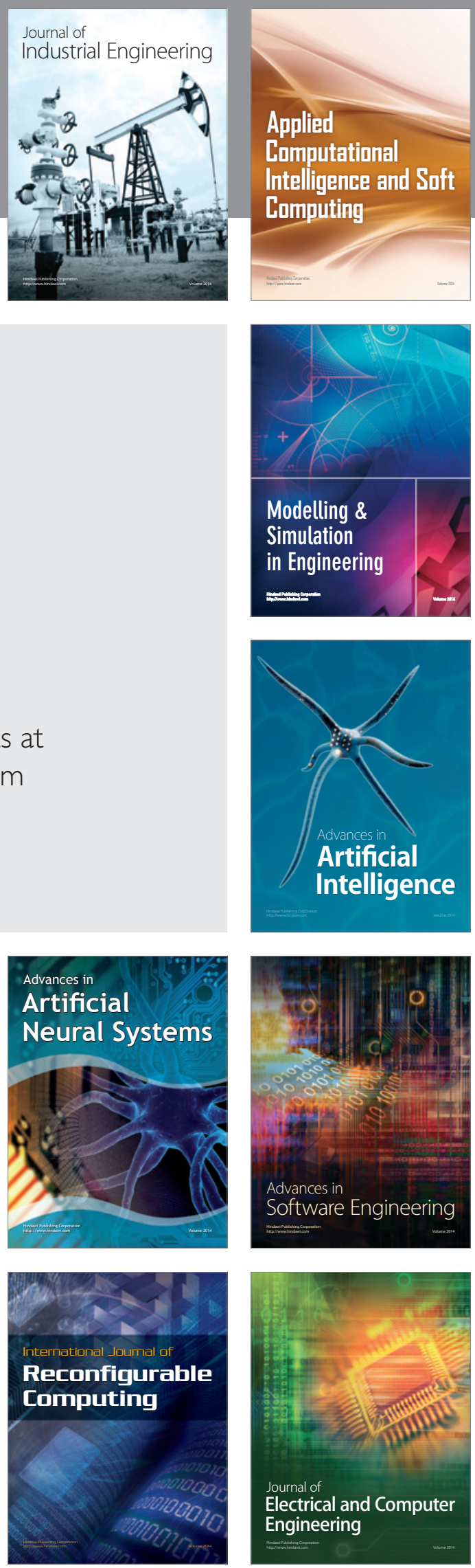